\title{
THE BICHROMATICITY OF A LATTICE-GRAPH
}

\author{
FRANK HARARY, DERBIAU HSU and ZEVI MILLER
}

(Received 1 July 1976)

Communicated by W. D. Wallis

\begin{abstract}
The bichromaticity $\beta(B)$ of a bipartite graph $B$ has been defined as the maximum order of a complete bipartite graph onto which $B$ is homomorphic. This number was previously determined for trees and even cycles. It is now shown that for a lattice-graph $P_{m} \times P_{n}$, the cartesian product of two paths, the bichromaticity is $2+\{m n / 2\}$.
\end{abstract}

The bichromaticity of a connected bipartite graph $B$, written $\beta(B)$, has been defined as the maximum order $p=r+s$ of a complete bigraph $K_{r . s}$ onto which $B$ is homomorphic, no two points of $B$ of different colors being sent to the same point. This is the invariant for bigraphs corresponding to the achromatic number of a graph $G$, defined by Harary and Hedetniemi (1970) as the maximum order of a complete graph onto which $G$ is homomorphic.

The majority of $B$ is the color class of maximum cardinality $\mu$ in $B$. It was shown by the present authors (1977) that for a tree, $\beta=1+\mu$ and for an even cycle $C_{2 n}, \beta=1+n$ if $n$ is odd and $2+n$ if $n$ is even.

The terminology and notation of the book of Harary (1969) will be used. The lattice-graph is the cartesian product $P_{m} \times P_{n}$ of two paths; it is obviously bipartite. We now develop a formula for its bichromaticity. To do this, we recall a basic but simple lemma proved in our previous paper.

LeMMA. If $h: B \rightarrow K_{r, s}$ is a bicomplete homomorphism of a noncomplete bigraph $B$ onto $K_{r, s}$ then $r s \leqq q$ and $r+s \geqq \mu+1$.

THEOREM. The bichromaticity of a lattice-graph is

$$
\beta\left(P_{m} \times P_{n}\right)=2+\{m n / 2\} .
$$

PRoof. For the sake of convenience, we first introduce some notation. Let $A=\{m n / 2\}$. View $P_{m} \times P_{n}$ as a lattice having $m$ rows and $n$ columns, where $v_{i j}$ is the point in row $i$ and column $j$. As a connected bigraph, $P_{m} \times P_{n}$ 
has the unique color classes $C$ and $D$, where without loss of generality $C$ contains all points $v_{i j}$ in the same color class as $v_{11}$ and $D$ all $v_{i j}$ in the same color class as $v_{12}$. Thus $|C|=A=\mu\left(P_{m} \times P_{n}\right)$ and $|D|=[m n / 2]$.

In order to show that the right side of (1) is a lower bound for $\beta\left(P_{m} \times P_{n}\right)$, we will define a bicomplete homomorphism $f: P_{m} \times P_{n} \rightarrow K(2, A)$ as follows. Let $X_{1}=\left\{v_{i j}: j\right.$ odd, $\left.v_{i j} \in D\right\}$ and $X_{2}=\left\{v_{i j}: j\right.$ even, $\left.v_{i j} \in D\right\}$, so $D$ may be partitioned $D=X_{1} \cup X_{2}$. To describe the action of $f$ on $C$, let all points in $X_{1}$ be sent by $f$ to one point $v$, and all points in $X_{2}$ to another point $w$. On the other hand, $f$ leaves all points of $C$ fixed. Then clearly $f\left(P_{m} \times P_{n}\right)=$ $K(2,\{m n / 2\})$, establishing the lower bound.

Our proof that the right side of (1) is also an upper bound for $\beta\left(P_{m} \times P_{n}\right)$,

$$
\beta\left(P_{m} \times P_{n}\right) \leqq 2+A,
$$

is considerably more involved. In order to accomplish this, it is sufficient to demonstrate the following proposition:

(P) If $h: P_{m} \times P_{n} \rightarrow K_{r, s}$ is a homomorphism for which $r+s=$ $\beta\left(P_{m} \times P_{n}\right)$, then $r \leqq 2$.

Once (P) has been verified, we have established (2) since $s \leqq \mu=A$. We begin by giving a simple argument that the possibilities $r=3$ and $r=4$ lead to contradictions. Later we shall see that $r \geqq 5$ is also impossible.

Assume first that $r=3$. The majority $C$ must contain at least two of the corner points of the lattice; call them $v$ and $w$. Each such point has degree 2 and its image under $h$ must have degree 3 since $r=3$. Therefore such a point is identified with at least one other point in $C$ under $h$. We may then have either $v$ and $w$ identified under $h$ with different points or with the same point. In both cases, the image of $C$ under $h$ will contain two fewer points than $C$, that is,

$$
s \leqq \overline{A-2} .
$$

Thus as a bound for $r+s$, we get

$$
r+s \leqq 3+A-2=1+A .
$$

But this contradicts $r+s=\beta\left(P_{m} \times P_{n}\right) \geqq 2+A$, as the lower bound in (1) is already verified.

Assume now that $r=4$. Since $s \geqq r=4$, the number of points in the lattice is at least 8 . Thus $C$ must contain at least three of the points on the "boundary" of the lattice. Each of these three points has degree at most 3 and the degree of its image under $h$ is 4 . Thus as above each of these three points must be identified with other points in $C$ by the homomorphism $h$. No matter how this is done, the image of $C$ under $h$ will contain at least 3 fewer points than $C$, so that 


$$
s \leqq A-3
$$

Again, we get as a bound

$$
r+s \leqq 4+A-3=A+1,
$$

contradicting $\beta\left(P_{m} \times P_{n}\right) \geqq 2+A$.

Having completed our consideration of the cases $r=3$ and 4 , we turn to an analysis of the remaining possibility $r \geqq 5$. First we show that any lattice satisfying the hypothesis of the lemma with $r \geqq 5$ has at most 72 points. Using this bound, it will then be shown that no such lattice can exist.

Applying the lemma to lattice-graphs, we have

$$
r s \leqq q\left(P_{m} \times P_{n}\right)=2 m n-m-n .
$$

Because we have already established $\beta\left(P_{m} \times P_{n}\right) \geqq 2+\{m n / 2\}$, we get

$$
r+s \geqq A+2 \geqq \frac{m n}{2}+2 \text {. }
$$

By combining inequalities (3) and (4), we find

$$
\frac{2 m n-m-n}{r}+r \geqq A+2 \geqq \frac{m n}{2}+2 \text {. }
$$

However, the usual inequality between the arithmetic mean and the geometric mean implies at once that $m+n \geqq \sqrt{2 m n}$. This inequality combined with the preceding one immediately gives

$$
r+\frac{2 m n-\sqrt{2 m n}}{r} \geqq \frac{m n}{2}+2 .
$$

For convenience, let us write $y=\sqrt{(m n)}$. It then follows directly that

$$
2 r^{2}-4 r \geqq(r-4) y^{2}+2 \sqrt{2 y} .
$$

We now discuss the problem in two cases: $r \geqq 6$ and $r=5$.

CASE 1. $r \geqq 6$. Here (5) gives

$$
2 r^{2}>2 r^{2}-24 \geqq 2 r^{2}-4 r \geqq y^{2}+2 \sqrt{2 y} \geqq y^{2},
$$

so that

$$
r>\frac{y}{\sqrt{2}}
$$

Furthermore

$$
r \leqq \frac{q\left(P_{m} \times P_{n}\right)}{S} \leqq \frac{q\left(P_{m} \times P_{n}\right)}{r}
$$


implies

This gives

$$
r^{2} \leqq 2 m n-m-n<2 m n=2 y^{2}
$$

$$
r \leqq \sqrt{2} y
$$

Combining this with (9), we find

$$
s+\sqrt{2} y \geqq r+s \geqq \frac{m n}{2}+2>\frac{y^{2}}{2} .
$$

Then we have

$$
s>\frac{y^{2}}{2}-\sqrt{2} y .
$$

But

$$
2 y^{2}=2 m n>2 m n-m-n \geqq r s>\left(\frac{y^{2}}{2}-\sqrt{2} y\right) \frac{y}{\sqrt{2}},
$$

where the last inequality follows from (6) and (7). Hence we get $\sqrt{m n}=y<$ $6 \sqrt{2}$, so that $m n<72$.

CASE 2. Suppose $r=5$. Then (5) gives $y<6$ so that $m n<36$.

Now Cases 1 and 2 have shown that under the hypothesis of $(P)$ with $r \geqq 5$, the lattice-graph $P_{m} \times P_{n}$ can have at most 72 points. As a first step in showing that such lattices cannot in fact exist, we prove that these lattices having $r \geqq 5$ must have at least 20 points. By the condition that $r \geqq 5$, there exists a homomorphism $h: P_{m} \times P_{n} \rightarrow K_{r, s}$ with $s \geqq r \geqq 5$ and $\beta\left(P_{m} \times P_{n}\right)=$ $r+s \geqq 10$. We now view $h$ as a sequence of elementary homomorphisms. Since $p\left(K_{r, s}\right) \geqq 10$ and each elementary homomorphism reduces the number of points in the lattice-graph by one, we see that $h$ is composed of a sequence of at most $m n-10$ elementary homomorphisms. We note further that since each elementary homomorphism fixes all but two points, the maximum number of points in $P_{m} \times P_{n}$ not fixed by $h$ is $2(m n-10)$. Now assume that $m n<20$ or in other words that $2(m n-10)<m n$. This means that at least one point $v$ of $P_{m} \times P_{n}$ is left fixed by $h$. Its degree in $h\left(P_{m} \times P_{n}\right)$ is then at most its degree in $P_{m} \times P_{n}$. That is,

$5 \leqq$ degree of $h(v)$ in $K_{r, s} \leqq$ degree of $v$ in $P_{m} \times P_{n} \leqq 4$.

a contradiction which shows that $m n \geqq 20$.

The next step will be to prove that a lattice satisfying the hypotheses of (P) with $r \geqq 5$ can have at most 16 points. This combined with $m n \geqq 20$ gives the final contradiction to $r \geqq 5$ and proposition $(\mathrm{P})$ will then be proved. Note 
first that as the maximum degree satisfies $\Delta\left(P_{m} \times P_{n}\right) \leqq 4$, each point of $C$ must experience at least one identification under $h$ if $r \geqq 5$. In fact, each point of $C$ must have as many identifications as the smallest multiple of 4 greater than or equal to $r$. Therefore

$$
s \leqq A /\{r / 4\}
$$

Now the conditions $m n<72$ and $r \geqq 5$ give $s \leqq 18$ as an upper bound. Furthermore if $r \geqq 13$, then $s \leqq 9$, contradicting $r \leqq s$. We thus conclude $5 \leqq r \leqq 12$.

We now get an upper bound for $m n$ in terms of $r$ by showing $m n \leqq 2(r-2) /(1-1 /\{r / 4\})$. Suppose this last inequality is false. Then using $r \geqq 5$ and manipulating this inequality routinely, we get

$$
r+A /\{r / 4\}<2+A \text {. }
$$

But by the above discussion, the left side is at least as large as $r+s$. Therefore

$$
r+s<2+A
$$

This contradicts $\beta\left(P_{m} \times P_{n}\right)=r+s$ since the lower bound $\beta\left(P_{m} \times P_{n}\right) \geqq$ $2+A$ has already been established.

As before, the homomorphism $h$ is composed of at most $m n-(r+s)$ elementary homomorphisms. Since $r+s \geqq 2 r$, we get

$$
m n-(r+s) \leqq 2((r-2) /(1-1 /\{r / 4\}))-2 r .
$$

Thus we have for an upper bound for the number of points not fixed by $h$,

$$
2(m n-(r+s)) \leqq 4((r-2) /(1-1 /\{r / 4\}))-4 r .
$$

The right side assumes the maximum 16 among the integers $r$ satisfying $5 \leqq r \leqq 12$. But this implies that if the lattice has more than 16 points, it has at least one fixed point under $h$. As observed previously, this contradicts the fact that each point in $h\left(P_{m} \times P_{n}\right)=K_{r, s}$ has degree at least 5. Therefore we conclude that if $r \geqq 5$, then $m n \leqq 16$, yielding the long awaited contradiction. The statement (P) is now proved and the theorem follows.

\section{Conclusion and unsolved problems}

1. For the cylinder $C_{2 n} \times P_{m}$ we have the following partial result. $\beta\left(C_{2 n} \times P_{m}\right)=\left\{\begin{array}{l}3+m n \text { if } 3 \mid n \text { or } 3 \times n \text { with } n \text { even and } m \text { odd } \\ 2+m n \text { or } 3+m n \text { otherwise }\end{array}\right.$

We conjecture that "otherwise" always yields $\beta=2+m n$. 
2. For the "torus-graph" $C_{2 n} \times C_{2 m}$ we have shown that $\beta\left(C_{2 n} \times C_{2 m}\right)=m n+i$, where $i=2$, 3, or 4 . However, we have not been successful in specifying the conditions which distinguish these three values.

We believe that a method for determining the bichromaticity of bigraphs may often be successfully developed in two stages. First, inequalities arising from the lemma may be used to give bounds on the parameters $r$ and $s$. Second, an ad hoc argument depending on the class of bigraphs in question will then yield an exact or "nearly" exact formula for the bichromaticity.

\section{References}

F. Harary (1969), Graph Theory (Addison-Wesley, Reading, Mass.)

F. Harary and S. T. Hedetniemi (1970), 'The achromatic number of a graph', J. Combinatorial Theory, 8, 154-161.

F. Harary, D. Hsu, and Z. Miller (1977), 'The bichromaticity of a tree', Theory and Applications of Graphs - in America's.Bicentennial Year. (Y. Alavi and D. R. Lick, eds., Springer-Verlag, Berlin.)

University of Michigan, Ann Arbor, Michigan, U.S.A. 